\title{
Minimizing convection effects to measure diffusion in liquid droplets during high-temperature electrostatic levitation
}

\author{
Sven Bossuyt \\ Vrije Universiteit Brussel (VUB), Department Mechanics of Materials and Constructions, B-1050 Brussels, \\ Belgium \\ Jan Schroers, Won-Kyu Rhim, and William L. Johnson \\ California Institute of Technology, Keck Laboratory of Engineering Materials 138-78, Pasadena, \\ California 91125
}

(Received 18 November 2004; accepted 12 January 2005; published online 2 March 2005)

\begin{abstract}
We present an approach to reduce the convective flow in an electrostatically levitated liquid droplet to such an extent that diffusion is the dominant mechanism for mass transport, thus enabling direct measurements of atomic diffusion in reactive liquids at elevated temperatures. Convection is minimized by containerless processing, and reducing temperature variations in the sample. The diffusion tracer is deposited in situ in the electrostatic levitation device used for containerless processing. Uniform noncontact heating of the sample is achieved by laser heating with multiple beams arranged symmetrically, e.g., in a tetrahedral geometry. The expected temperature variations and the resulting convection flows are estimated for a Zr-based glass-forming alloy. The analysis suggests that diffusion experiments are possible throughout the entire undercooled liquid temperature range of this alloy and, in microgravity, up to $50 \mathrm{~K}$ above the liquidus temperature. (C) 2005 American Institute of Physics. [DOI: 10.1063/1.1866932]
\end{abstract}

\section{INTRODUCTION}

As a platform for experiments on reactive materials at high-temperature, electrostatic levitation (ESL) is in many ways ideal. Because ESL is a containerless processing method operated in ultrahigh vacuum, the sample is almost perfectly isolated from its environment. The levitation mechanism is conceptually simple, despite the experimental complications of an active control system required to maintain a stationary sample position. ${ }^{1,2}$ Although limited to noncontact methods, a wide variety of thermophysical properties have been measured in ESL experiments, including heat capacity and hemispherical emissivity, ${ }^{3-5}$ phase transition temperatures, ${ }^{4,6,7}$ density and thermal expansion, ${ }^{5,8,9}$ viscosity and surface tension, ${ }^{5,10}$ electrical conductivity, ${ }^{11}$ and x-ray or neutron scattering structure factors. ${ }^{12,13}$ To further expand the electrostatic levitation technique and enable the measurement of transport properties of liquids, the contribution from convective transport must be either negligible or precisely known.

The present paper presents a systematic analysisbuilding on the earlier work of Bauer ${ }^{14,15}$ of the relative magnitude of the different driving forces for convection in an ESL experiment, and establishes the boundaries within which mass transport is dominated by diffusion. Gravitational body forces (natural convection) and variations in surface tension (Marangoni convection) that arise from thermal gradients and solute concentration gradients are considered. The calculations show that convection can be suppressed to an acceptable level even at temperatures above the liquidus, when the experiment is performed using a tetrahedral heating assembly. We demonstrate with data for the bulk glass form- ing $\mathrm{Zr}_{58} \mathrm{Nb}_{3} \mathrm{Cu}_{16} \mathrm{Ni}_{13} \mathrm{Al}_{10}$ that measurement of atomic diffusion over the entire undercooled liquid region then becomes feasible, limited only by practicalities related to sample processing and the diffusion measurement itself. Thus, we arrive at an approach to measuring atomic transport in chemically reactive high-temperature liquids, which overcomes problems associated with convection and reactivity.

\section{ANALYSIS AND MODELING OF CONVECTION EFFECTS}

With high-vacuum containerless processing, only a limited number of interactions of a sample with its environment are a priori possible. The sample is almost perfectly isolated. This has the disadvantage that all measurements and manipulations must be made by noncontact methods, but analysis and modeling is conveniently simplified. The only forces acting on the sample are: external forces of gravity and electromagnetism, internal forces governing fluid flow as described by the Navier-Stokes equations, and surface tension at the liquid-vacuum interface. To evaluate the resulting convective flow velocities, one might use several approaches.

The simplest approach is a dimensional analysis, for each of the different driving forces for convection, to determine the influence of the various parameters that affect convection and calculate the order of magnitude of the induced convective flow velocity. Dimensionless numbers combine the properties of the liquid (viscosity, heat capacity, density, surface tension, etc.), the applied boundary conditions (temperature, solute flux, length scale), and the magnitude of the resulting flow velocity, so that the problem can be modeled by a set of functions which depend only on these dimensionless numbers and the geometry of the problem. Even without 


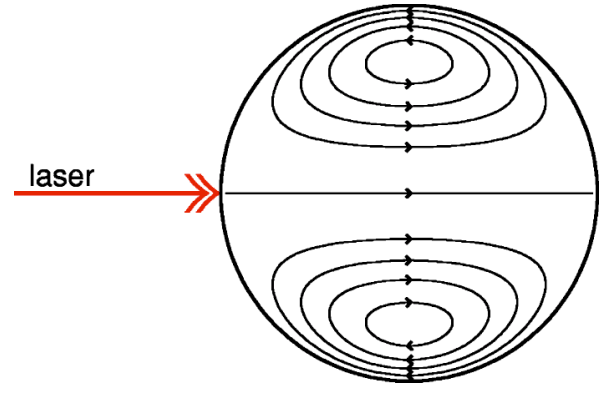

FIG. 1. (Color online) Schematic illustration of Marangoni flow field for a sphere subjected to constant temperature gradient. This is the leading term in the flow field induced by unidirectional heating with a laser beam.

solving the actual functions giving numerical values for the quantities of interest, much useful information can be extracted from the dimensionless numbers that appear in the problem.

A more quantitative assessment can be obtained by examining known analytical solutions to the Navier-Stokes equations, where such solutions exist, or going to numerical solutions. The following discussion gives results from dimensional analysis and approximate analytical solutions for the limiting case where convective flow velocities remain small.

\section{A. Marangoni convection}

Bauer $^{15}$ has solved the linearized Navier-Stokes equation for the case of a freely floating sphere subjected to an arbitrary axially symmetric thermal gradient and temperature-dependent surface tension, to obtain a series expansion for the analytical solutions of the stream function for thermally induced Marangoni convection

$$
\begin{aligned}
u_{M}(r, \theta)= & \frac{1}{2 \eta}\left|\frac{d \sigma}{d T}\right| \sum_{n=1}^{\infty} T_{n} \frac{n(n-1)}{2 n+1}\left(\left(\frac{r}{R}\right)^{n-1}\right. \\
& \left.-\left(\frac{r}{R}\right)^{n+1}\right) P_{n}(\cos (\theta)), \\
v_{M}(r, \theta)= & \frac{1}{2 \eta}\left|\frac{d \sigma}{d T}\right| \sum_{n=1}^{\infty} T_{n} \frac{1}{2 n+1}\left((n+1)\left(\frac{r}{R}\right)^{n-1}\right. \\
& \left.-(n+3)\left(\frac{r}{R}\right)^{n+1}\right) P_{n}^{1}(\cos (\theta)),
\end{aligned}
$$

where $u_{M}$ and $v_{M}$ are the radial and tangential components of the flow velocity, expressed in polar coordinates $r$ and $\theta, \eta$ is the viscosity, $d \sigma / d T$ is the temperature dependence of the liquid surface tension, $R$ is the radius of the sphere, and $T_{n}$ are the coefficients in a Legendre polynomial series expansion of temperature (see Sec. II C below), with $P_{n}(\xi)$ and $P_{n}^{1}(\xi)$ the unassociated and associated Legendre polynomials, respectively. The subscript " $M$ " refers to Marangoni driven flow. Because the equations are linearized, solutions may be superimposed, so that the series expansion is readily generalized to a multipole expansion relaxing the axial symmetry requirement. Figure 1 illustrates the steady-state flow field for the leading term in the series expansion, i.e., in the case of a constant temperature gradient. The flow field is toroidal, with the axis of symmetry along the temperature gradient

$$
\begin{aligned}
& u_{M}(r, \theta)=(\Delta T / 3 \eta)|d \sigma / d T|\left\{1-r^{2} / R^{2}\right\} \cos \theta, \\
& v_{M}(r, \theta)=-(\Delta T / 3 \eta)|d \sigma / d T|\left\{1-2 r^{2} / R^{2}\right\} \sin \theta,
\end{aligned}
$$

where $\Delta T$ is the temperature difference between the poles of the sphere. The maximum flow velocity, with magnitude $v_{\max , M}=(\Delta T / 3 \eta)|d \sigma / d T|$, occurs tangentially at the equator $(\theta=\pi / 2)$ on the sphere surface $(r=R)$ and also along the polar axis $(\theta=0$ or $\theta=\pi)$ at the sphere center $(r=0)$. Note that whereas the radial component always vanishes at the surface, the tangential component is nonvanishing owing to the surface tractions presented by the thermally varying surface tension. For higher-order terms in Eq. (1), the corresponding flow field lines separate into several lobes displaying the symmetry characteristic of that multipole term. Taking the maximum flow velocity for each term in the multipole expansion separately, the position of the maximum is found nearer the surface of the sphere for higher order terms, but the magnitude is always of the order of $v_{\max , M}$, where $\Delta T$ is replaced with the magnitude of the corresponding term in the multipole expansion for temperature. ${ }^{16}$

\section{B. Buoyancy-driven convection}

Due to thermal expansion, temperature differences are associated with differences in density, which are the cause of natural (or free, as opposed to forced) convection. Bauer has extended his analytical solution for Marangoni convection in a free-floating sphere, given in Eq. (1), to also include the effect of gravity. ${ }^{14}$ The flow field can be calculated for the combined effects of buoyancy in a constant gravitation field and Marangoni convection with a temperature field that is axially symmetric about an axis aligned with the direction of gravity. Using this analytical solution, the relative magnitudes and combined influences of Marangoni and gravitational flow as a function of sample size, temperature, temperature gradient, magnitude of the gravitation field, and other relevant parameters can be assessed. The ratio of driving forces for buoyancy-driven and Marangoni convection is given by the length scale dependent dynamic Bond number, ${ }^{17}$ defined as

$$
B o[L]=g \rho \beta L^{2} /[d \sigma / d T],
$$

where $g$ is the gravitational acceleration, $\rho$ the density, $\beta$ the volume thermal expansion coefficient, and $L$ the characteristic length scale of the density variation.

\section{Temperature gradients}

The magnitude of temperature variations expected in the spherical droplets under conditions of heating with one or more laser beams (or any other power input source) can be assessed by solving the Fourier heat flow equation. The boundary conditions represent heat loss from the surface by Stephan-Boltzmann radiation to a surrounding vacuum and heat input by absorption of laser power

$$
J_{r}(R, \theta, \varphi)=-\kappa[\partial T / \partial r]_{r=R}=\varepsilon \sigma_{\mathrm{SB}} T_{s}^{4}-p_{\text {laser }}(\theta, \varphi),
$$

where $J_{r}(R, \theta, \varphi)$ is the net radial component of the heat flux out of the sphere surface, $\kappa$ is the thermal conductivity of the 


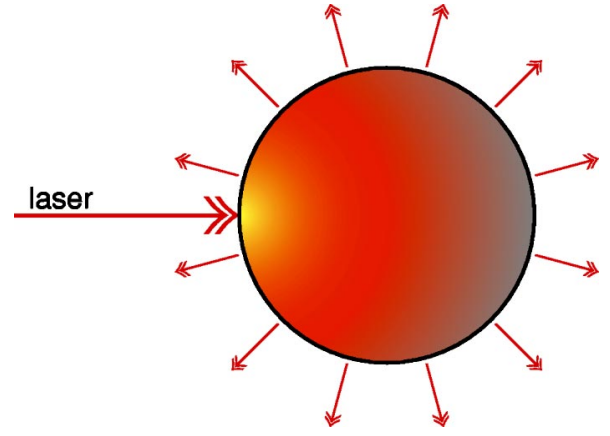

FIG. 2. (Color online) Schematic illustration of single beam heating by a laser beam and radiation loss by the sphere producing a temperature gradient.

liquid, $T_{s}$ is the local surface temperature, $\varepsilon$ is the total hemispherical emissivity of the surface, $\sigma_{\mathrm{SB}}$ the StephanBoltzmann constant, and $p_{\text {laser }}$ the laser power input at the sphere surface, which depends on location $(\theta, \varphi)$. The power input is determined by the spectral absorption coefficient of the surface (equal to the spectral emissivity) at the laser wavelength and the incident power per unit area of the laser beam projected on the sphere surface. The situation is illustrated in Fig. 2. For small temperature gradients, such that $\Delta T / T_{\text {av }} \ll 1$, the local temperature $T_{s}$ in Eq. (4) may be replaced by the average surface temperature of the sphere $T_{\mathrm{av}}$, to a good approximation. ${ }^{18}$

The steady-state solution can be expressed as a multipole series expansion using spherical harmonics; for axially symmetric problems these reduce to Legendre polynomials, as used in the analytical solutions for Marangoni and gravitydriven convection ${ }^{14,15}$

$$
T(r, \theta, \varphi)=\sum_{n=0}^{\infty} T_{n} \frac{r^{n}}{R^{n}} P_{n}(\cos (\theta))
$$

with

$$
\begin{aligned}
T_{n}= & \left(1+\frac{1}{2 n}\right) \frac{R}{\kappa} \int_{0}^{\pi / 2} \cos (\theta) \varepsilon I_{\text {laser }}(R \sin (\theta)) \\
& \times P_{n}(\cos (\theta)) \sin (\theta) d \theta \text { for } n>0
\end{aligned}
$$

and

$$
T_{0}=\sqrt[4]{\left(\int_{0}^{\pi / 2} \cos (\theta) \varepsilon I_{\text {laser }}(R \sin (\theta)) \sin (\theta) d \theta / 2 \varepsilon \sigma_{\mathrm{SB}}\right)}
$$

for an axially symmetric laser beam centered on a spherical sample, with intensity profile $I_{\text {laser }}(x)$ in $\mathrm{W} / \mathrm{m}^{2}$. For multiple laser beams, the average temperature $T_{0}$ must be calculated using the total power input from all of the beams together, but the rest of the temperature profile may be calculated for each beam separately and superimposed. In any case, the Marangoni flow velocity profile scales as

$$
v_{\text {max }, M} \propto R p_{\text {laser }}|d \sigma / d T| / \kappa \propto R \sigma_{\mathrm{SB}} T_{\mathrm{av}}^{4}|d \sigma / d T| / \kappa .
$$

\section{Composition variations}

To assess the role of composition variations in inducing convective flow (solutal convection), one needs to account for the fact that both the density and the surface tension of the alloy are composition dependent and may therefore vary with solute concentration. We can estimate the driving forces for Marangoni solutal convection by referring to the previous discussion [Eqs. (1) and (6)] and replacing the thermal driving force for Marangoni convection $\nabla T[d \sigma / d T]$ by the equivalent solutal driving force $\nabla c[d \sigma / d c]$. Here $c$ is the dimensionless concentration of solute $(c=1$ for a pure solute phase), $\nabla c$ is the magnitude of the concentration gradients on the sample surface, and $d \sigma / d c$ is the concentration dependence of the liquid surface tension. We can define a dimensionless ratio

$$
\delta=\nabla c[d \sigma / d c] / \Delta T[d \sigma / d T]
$$

which measures the ratio of solutal to thermal driving forces. In principle, the solutal driving force for Marangoni convection could be much larger than the thermal driving force. To estimate the composition dependence of the surface tension of an alloy, one should remember that alloys minimize their surface energy by surface segregation of the element(s) with lowest surface tension. As a result, the surface tension changes dramatically with the concentration of these low surface tension elements when their concentration is low, but when the surface tension of the alloy approaches the surface tension of the low surface tension element it is relatively independent of composition. Often the solute does not lower surface tension of the alloy, and $d \sigma / d c \ll \sigma$ for all concentrations. In addition, surface diffusion-which is much faster than bulk diffusion-may well override solutal Marangoni convection as a mechanism for reducing concentration variations on the surface. It is therefore likely that solutal Marangoni convection would be insignificant.

Concentration variations in the sample can also cause buoyancy-driven convection. We can again replace the thermal driving force $g \rho \beta \Delta T$ in Eq. (3) with the corresponding solutal driving force $g[d \rho / d c] \Delta c$, where $d \rho / d c$ is the concentration dependence of the liquid density, and $\Delta c$ is the magnitude of the concentration variations within the sample. Or we can define a dimensionless ratio

$$
\gamma=\Delta c[d \rho / d c] / \rho \beta \Delta T
$$

to measure the ratio of solutal to thermal driving forces for natural buoyancy-driven convection.

\section{E. Dynamic effects}

So far, the analysis considered steady-state solutions, with driving forces for convection that are constant over time. The steady-state solutions are reached only when transients resulting from previous changes and disturbances have decayed. For convection, viscous dissipation gives a damping time of

$$
t_{\mathrm{visc}}=\rho L^{2} / \eta,
$$

where $L$ is a transverse length scale of the transient flow pattern. For changing temperature, the internal thermal relaxation time-governing the decay of temperature variations within the sample-is given by 


$$
t_{\text {therm }}=c_{P} R^{2} / \pi^{2} \kappa,
$$

whereas the external thermal relaxation time-required for the average sample temperature to adjust to changes in net heating power-is

$$
t_{\mathrm{rad}}=R c_{P}\left(T_{0}-T_{\mathrm{RT}}\right) / \varepsilon \sigma_{\mathrm{SB}}\left(T_{0}^{4}-T_{\mathrm{RT}}^{4}\right) .
$$

Here $c_{P}$ is the specific heat capacity per unit volume of the sample material, $\kappa$ is its thermal conductivity, and $T_{\mathrm{RT}}$ is the ambient temperature.

It is also possible that the sample would rotate. This can be controlled in the electrostatic levitation experiment, by adjusting one of the laser beams so its incidence on the sample is off-center. The moment of the momentum of the absorbed photons is then converted into angular momentum of the sample, gradually increasing or decreasing its rotation rate, with a rotational acceleration of up to $0.05 \mathrm{~Hz} / \mathrm{s}$. ${ }^{19}$ The effect of a rotating reference frame on the kinematics of convection is accounted for by augmenting body forces with centrifugal and Coriolis "forces." For slow rotations and vanishing convection velocities (i.e., nearly rigid-body rotation), both effects can be neglected.

\section{MINIMIZING CONVECTION}

The analysis and modeling results indicate practical methods for reducing convective flow to an acceptable minimum. Depending on the situation, different measures of the convective flow velocity profile determine what is acceptable: total displacement of a material point, convective flow velocity, local shear rate, etc. For each of these quantities the maximum or the average over time and/or space, its magnitude or the component along some direction, over the entire sample or only a part of it may be the most relevant. In most cases, reducing one measure of the flow velocity profile will also reduce the others, so we may simply state the goal as minimizing convection. Which combination of the methods below should be used will be determined by the expense and practicality of implementing each method under the circumstances dictated by the experiment, and by the sensitivity of the experimental outcomes to disturbance by convective flow.

\section{A. Improved temperature uniformity}

Both Marangoni convection and thermal buoyancydriven convection are caused by temperature variations in the sample. The magnitude of these variations can be drastically reduced by using a symmetric arrangement of broad laser beams instead of a single narrow beam for heating. ${ }^{18}$ In fact, the limiting case of an infinitely narrow beam is the worst possible case for temperature uniformity: heat flux and temperature at the point of incidence become infinite, and the Legendre series expansions of Eqs. (1) and (5) cannot be truncated. If the power input is distributed more smoothly over the surface, without any sharp features, the higher-order terms in the Legendre series are not needed to provide a good description of the power input and the resulting temperature and flow fields. The symmetry of the Legendre polynomials further simplifies the series expansion when a symmetric arrangement of multiple heating laser beams is used. Figure 3 illustrates that increasing the beam width reduces the higher-order terms disproportionately, while symmetry eliminates the lowest-order terms.

The complementary effects of symmetry and beam broadening in the heating arrangement are most visible in different parts of the convective flow velocity profile. Convective flow near the center of the sample is caused by temperature differences across the sample, and therefore most effectively reduced by using a more symmetric heating arrangement. Precisely balancing the power and alignment of the different heating beams is paramount. Sharp features in the heating power distribution are associated with convective eddy flows localized beneath these features near the sample surface. These are most effectively reduced by smoothing and broadening the heating beam intensity profile.

\section{B. Controlled sample rotation}

In a rotating sample, centrifugal acceleration and gyroscopic forces can complicate matters, causing significant deviations from the nominally spherical sample shape at high rotation rates, ultimately leading to dynamic instabilities. The rotation rate must be kept low enough to avoid these effects. But rotation also has the effect of smoothing out azimuthal variations in any external fluxes incident on the sample, e.g., the power input from the heating laser beam(s). Provided that rigid body rotation does not run counter to other experimental requirements, it may therefore be desirable to work with the sample spinning at a rate $\psi$ such that

$$
t_{\text {relax }} \gg \omega^{-1}>\left(g_{\text {max }} / R\right)^{-1 / 2},
$$

where $t_{\text {relax }}$ is the internal relaxation time for the variations in the sample caused by the nonuniform flux, $g_{\max }$ is the allowable centrifugal acceleration, and $R$ is the sample radius. Deviations from the spherical sample shape remain small when the pressure of the centrifugal force is small compared to the surface tension $\sigma$,

$$
\rho \omega^{2} R^{3} / \sigma \ll 1 \text {. }
$$

\section{Microgravity environment}

An obvious, but somewhat impractical and very expensive, method of reducing buoyancy-driven convection is to reduce gravity. Due to the existence of research laboratories in parabolic flight or low earth orbit, made available by government space programs, carrying out electrostatic levitation experiments in microgravity might be an option. In a microgravity environment, the hydrostatic pressure gradient in the sample is reduced by several orders of magnitude. This pressure gradient causes buoyancy, driving convection, and deviations from the perfectly spherical sample shape favored by surface tension. The electric field strength required to levitate a given sample, which is another source of shape distortions and limits the sample size that may be levitated, is also reduced proportionally to gravity.

\section{Sample material properties}

When different sample materials are under consideration for an experiment, the choice can be partly determined by the 

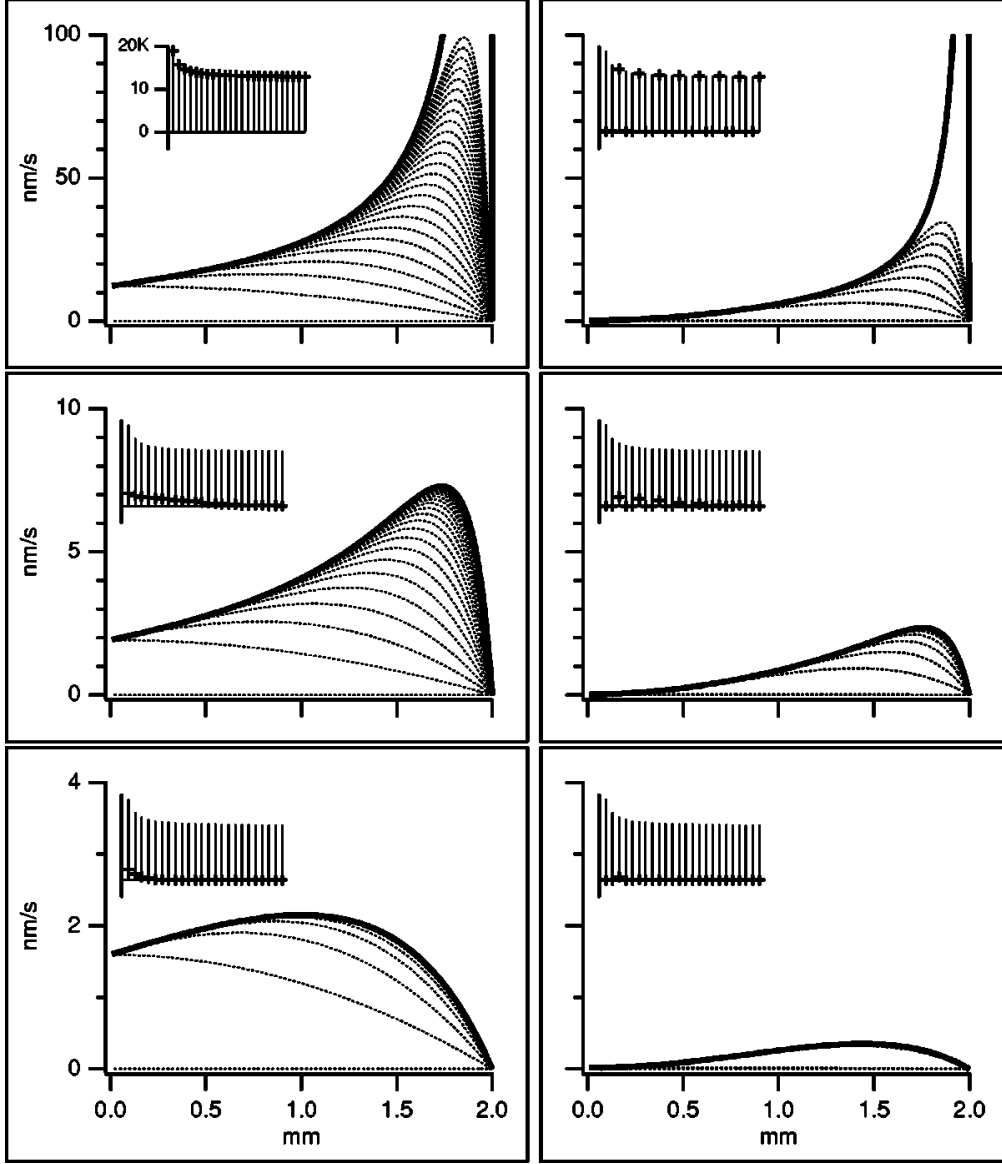

FIG. 3. Radial component of Marangoni flow velocity plotted versus position along the polar axis, calculated according to Eq. (1), for different heating beam configurations: limiting case of infinitely narrow laser beam (top row), typical heating laser beam with Gaussian intensity profile of $0.5 \mathrm{~mm}$ FWHM (middle row), and heating laser beam with Gaussian intensity profile broadened to $2 \mathrm{~mm}$ FWHM (bottom row), in each case for a single laser beam (left column) and 4 laser beams in a tetrahedral geometry (right column). The thin dotted lines delineate the contributions of the first 25 terms of the summation in Eq. (1), and the inset graph shows the corresponding coefficients $T_{n}$. The calculations were carried out using material properties listed in Table I for a sphere of $4 \mathrm{~mm}$ diameter at $960 \mathrm{~K}$, with a $1 \%$ allowance for imperfect alignment and beam intensity calibration in the tetrahedral geometry. materials' physical properties and the role these play in the various contributions to convective flow. For the same driving forces and geometry, material with a higher viscosity would have proportionally lower flow velocities. The different driving forces and the parameters that determine the experiment duration and temperature should also be evaluated for each material.

The buoyancy-driven contribution to convective flow is directly proportional to the density difference between different parts of the sample. Density differences can result from phase changes, temperature differences or composition differences. When different sample materials are under consideration for an experiment, a choice can be made to avoid excessive buoyancy-driven convection.

\section{CASE STUDY}

In the following, the analysis of convective flow will be applied to evaluate the feasibility of diffusion measurements on the melt of a bulk glass forming alloy, using an electrostatic levitation technique for sample containment. The high viscosity and good thermal stability of bulk glass-forming alloys in the deeply undercooled liquid state have enabled several diffusion studies since the discovery of these alloys. ${ }^{20-22}$ We shall not concern ourselves with the accuracy of our assumptions or the feasibility of the electrostatic levitation approach near the glass transition, but at higher temperatures where a containerless technique is needed to avoid chemical reactions between the container wall and the liquid.
Convective flow must be avoided as well. Convective mixing can completely obliterate the diffusion profile; even small amounts of convection contaminate the profile and make quantitative analysis difficult or impossible. In the past, researchers have employed capillary geometries, magnetic damping, and reduced gravity to limit the contamination of diffusion profiles by convection. ${ }^{23,24}$ Nearly all of the earlier research on diffusion in metallic liquids has been confined to low melting point metals such as indium, gallium, tin, bismuth, and similar alloys. One reason for this is that, at elevated temperature, temperature gradients - and the resulting Marangoni- and gravity-driven (natural) convective flows - tend to become aggravated. In simulations it was found that contamination in capillary experiments remains a serious problem. ${ }^{25,26}$ Experiments performed under microgravity conditions on low melting point liquids have shown that, in some cases, problems associated with natural convection can be overcome. ${ }^{27}$ As a benchmark for the utility of a proposed method of reducing convection in liquids, a feasibility study of high-temperature diffusion measurements seems appropriate.

\section{A. Alloy properties}

We chose $\mathrm{Zr}_{58} \mathrm{Nb}_{3} \mathrm{Cu}_{16} \mathrm{Ni}_{13} \mathrm{Al}_{10}$ (Vit106a), which is the best currently known Zr-based metallic glass forming composition without beryllium. ${ }^{28}$ The physical properties of this alloy - or related alloys when data for this composition was unavailable_-are listed in Table I. Recently, it was shown that the Vit106a alloy could be vitrified by radiative cooling. 
TABLE I. Values of various physical parameters used for the calculations. For the cases where parameters are not available for Vit106a $\left(\mathrm{Zr}_{58} \mathrm{Nb}_{3} \mathrm{Cu}_{16} \mathrm{Ni}_{13} \mathrm{Al}_{10}\right.$ ), we chose the parameters for the neighboring composition of Vit106 $\left(\mathrm{Zr}_{57} \mathrm{Nb}_{5} \mathrm{Cu}_{15} \mathrm{Ni}_{13} \mathrm{Al}_{10}\right)$.

\begin{tabular}{lc}
\hline \hline \multicolumn{1}{c}{ Physical parameter } & Value \\
\hline Molar mass $^{2}$ & $W_{\text {mol }}=0.07617 \mathrm{~kg} / \mathrm{mol}$ \\
Density $^{\mathrm{a}}$ & $\rho=6.66 \times 10^{3} \mathrm{~kg} / \mathrm{m}^{3}$ \\
Thermal expansion $^{\mathrm{a}}$ & $\beta=5.33 \times 10^{-5} \mathrm{~K}^{-1}$ \\
Glass transition temperature $^{\mathrm{b}}$ & $T_{g}=673 \mathrm{~K}$ \\
Liquidus temperature $^{\mathrm{b}}$ & $T_{\mathrm{liq}}=1103 \mathrm{~K}$ \\
Specific heat $^{\mathrm{c}}$ & $c_{p}=3.9 \times 10^{6} \mathrm{~J} / \mathrm{m}^{3} \mathrm{~K}$ \\
Thermal conductivity $^{\mathrm{d}}$ & $\kappa \sim 25 \mathrm{~W} / \mathrm{m} \mathrm{K}^{-6}$ \\
& $\alpha=\kappa / c_{p} \sim 6 \times 10^{-6} \mathrm{~m}^{2} / \mathrm{s}$ \\
& $\varepsilon=0.26$ \\
Total hemispherical emissivity $^{\mathrm{d}}$ & $\varepsilon=0.26$ \\
Spectral emissivity $^{\mathrm{d}}$ & $\sigma=\left(1.538-1.7 \times 10^{-5} \mathrm{~T}\right) \mathrm{J} / \mathrm{m}^{2}$ \\
Surface tension $^{\mathrm{e}}$ &
\end{tabular}

${ }^{\mathrm{a}}$ Data for Vit106 at $T_{\text {liq }}$, from Ref. 30.

${ }^{\mathrm{b}}$ Data from Ref. 29.

${ }^{\mathrm{c}}$ Data for Vit106 at $T_{\text {liq, }}$, from Ref. 32 .

${ }^{\mathrm{d}}$ Data for Vit106, from Ref. 33.

${ }^{\mathrm{e}}$ Data for Vit106 with $T$ in K, from Ref. 34.

Isothermal crystallization studies were performed in the entire undercooled liquid region and compiled in a timetemperature-transformation (TTT) diagram. ${ }^{29}$ The TTTdiagram has a typical nose shape, with the shortest time of $30 \mathrm{~s}$ to crystallization occurring at $900 \mathrm{~K}$. We will consider three different temperatures, representative of the entire undercooled liquid region: $960 \mathrm{~K}$, above the nose of the TTTdiagram, $900 \mathrm{~K}$, at the nose, and $740 \mathrm{~K}$, below the nose.

For the further calculations, the viscosity and diffusivity and their temperature dependence are required. For the Vit106a alloy, this data has not been measured yet. The viscosity can be estimated using data for the viscosity of the original Vit106 composition, ${ }^{30}$ which is very similar to Vit106a, interpolated to the temperatures of interest using the Vogel-Fulcher-Tamman equation. If the temperature axis is slightly adjusted to move liquidus and glass transition temperatures of Vit106 to those of Vit106a, the viscosity of Vit106a is estimated to be $10 \mathrm{kPa} \mathrm{s}$ at $960 \mathrm{~K}, 75 \mathrm{kPa} \mathrm{s}$ at $900 \mathrm{~K}$, and $5 \mathrm{GPa}$ s at $740 \mathrm{~K}$. For the diffusivity, we assume a typical value for elemental metallic melts at about $1000 \mathrm{~K}$ of $D=3 \times 10^{-9} \mathrm{~m}^{2} / \mathrm{s}$ and further assume that-far above the glass transition temperature-the Einstein-Stokes relationship holds to estimate its temperature dependence. ${ }^{31}$ This results in diffusivity values of approximately $1 \times 10^{-10} \mathrm{~m}^{2} / \mathrm{s}$ at $960 \mathrm{~K}, 1 \times 10^{-11} \mathrm{~m}^{2} / \mathrm{s}$ at $900 \mathrm{~K}$, and $1 \times 10^{-16} \mathrm{~m}^{2} / \mathrm{s}$ at $740 \mathrm{~K}$.

\section{B. Tracer deposition}

In order to perform a diffusion experiment, a "tracer" species will be deployed on the surface of the molten sphere by vapor deposition. Treating diffusivity as a constant, the evolution of the (dilute) tracer diffusion profile in the absence of convection can be calculated analytically. If the tracer deposition is uniform across the sample surface with a constant flux $F_{0}$,

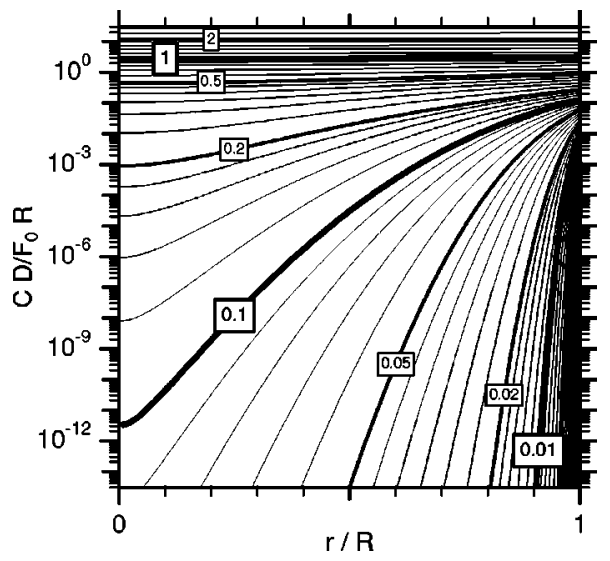

FIG. 4. Graph of diffusion profiles labeled with the corresponding dimensionless diffusion length $(D t)^{1 / 2} / R$.

$$
\begin{aligned}
C(t, r)= & \frac{F_{0} R}{D}\left\{3 \frac{D t}{R^{2}}+\frac{1}{2}\left(\frac{r}{R}\right)^{2}-\frac{3}{10}\right. \\
& \left.-2 \frac{R}{r} \sum_{n=1}^{\infty} \frac{\sin \left(\alpha_{n} r / R\right)}{\alpha_{n}^{2} \sin \left(\alpha_{n}\right)} \exp \left(-\alpha_{n}^{2} \frac{D t}{R^{2}}\right)\right\}
\end{aligned}
$$

with $\alpha_{n}$ the $n$th positive root of $\alpha_{n}=\tan \left(\alpha_{n}\right)$. For small values of the diffusion length $\lambda=(D t)^{1 / 2}$ compared to the sample radius $R$, the profile can be approximated by writing the analytical solution for continuous deposition on a planar surface in spherical coordinates

$$
\begin{aligned}
C(t, r)= & \frac{F_{0} R}{D}\left\{\sqrt{\frac{4 D t}{\pi r^{2}}} e^{-\left[(R-r)^{2} / 4 D t\right]}\right. \\
& \left.-\frac{R-r}{r} \operatorname{erfc}\left(\frac{R-r}{\sqrt{4 D t}}\right)\right\},
\end{aligned}
$$

where $\operatorname{erfc}(x)$ is the complementary error function. Dimensionless concentration profiles are plotted for different values of the ratio $\lambda / R$ in Fig. 4.

Vapor deposition sources like Knudsen cells are capable of achieving a deposition rate of $10^{-11} \mathrm{~m} / \mathrm{s}$. An alternative way to apply the tracer on the sample surface is chemical vapor deposition. Filling the chamber with a suitable deployment gas to a low pressure of about $10^{-6}$ mbar results in a uniform deployment of the tracer on the sample's surface, with a deposition rate of about one monolayer per second. Several deployment gases are available to study the diffusion of the alloy's elements.

A meaningful analysis of the diffusion profile requires the diffusion length, $\lambda$, to be significantly smaller than the sample radius to avoid reaching a nearly uniform distribution of the tracer species. On the other hand, the profile must be at least $30 \mathrm{~nm}$ deep in order to be evaluated by a suitable method such as secondary ion mass spectroscopy (SIMS). In an ideal profile, concentrations span the entire range from the detection limit of the analysis technique to the maximum concentration that is still dilute, i.e., which does not noticeably affect the outcome of the diffusion experiment. Figure 4 demonstrates that diffusion profiles with $\lambda \approx 0.1 R$ or smaller span many orders of magnitude in concentration. 
The maximum concentration that can still be considered dilute depends on which physical properties of the liquid are of importance in the diffusion experiment, and how strongly those depend on the solute concentration. In any case, the dilute limit will be below about $1 \%$, but for some tracer species, the limit can be much lower. With "heavy" elements like $\mathrm{Au}$, for example, $d \rho / d c$ is of the order of $10^{4} \mathrm{~kg} / \mathrm{m}^{3}$, which gives a maximum concentration of about $10 \mathrm{ppm}$ to keep the dimensionless ratio $\gamma$ in Eq. (8) smaller than one.

\section{Perturbation of the diffusion profile by convection}

To assess the degree to which experimentally determined atomic concentration profiles are contaminated by the effects of convection, the rate of atomic transport by diffusion should be compared with the rate of convective transport. Strictly speaking, the relevant quantity is the total change of the atomic concentration, i.e., the time integral of the divergence of the concentration flux vectors

$$
C\left(t_{1}\right)-C\left(t_{0}\right)=\int_{t_{0}}^{t_{1}} \frac{d C}{d t} d t=\int_{t_{0}}^{t_{1}} \bar{\nabla} \cdot(D \bar{\nabla} C)-\bar{v} \cdot \bar{\nabla} C d t,
$$

where $C(t)$ is the concentration at time $t, D$ is the intrinsic atomic diffusion constant, and $v$ is the convective flow velocity. The first term in the integral is associated with Fick's law of diffusion, whereas the second term represents convection. If, at all times, everywhere in the sample, the convection term is negligible compared to the diffusion term, a diffusion profile uncontaminated by convection is guaranteed. Since we are primarily interested in the limiting case where the perturbations remain negligible, a first-order approximation may be used, where the convection and diffusion problems are solved independently, combining the convective flow profile with the unperturbed diffusion profile to calculate the perturbation of the concentration profile.

For comparison of diffusion with convection, it is instructive to define a diffusion velocity $v_{\text {diff }}$ such that the "convective" flux associated with this "velocity" equals the diffusion flux

$$
v_{\mathrm{diff}}=\frac{\bar{\nabla} \cdot(D \bar{\nabla} C)}{\|\bar{\nabla} C\|^{2}} \bar{\nabla} C .
$$

For the solution given in Eq. (14), the diffusion velocity is a monotonically decreasing function of time, of the order of $(D / \pi t)^{1 / 2}$ in the initial stages-where the diffusion length $\lambda=(D t)^{1 / 2}$ is small - and approaching $3 D / r$ when the diffusion length becomes large compared to the sample radius. In order to keep the diffusion profile free of convective perturbation, it is sufficient that $v_{\text {diff }} \gg v_{\max , M}$ and $v_{\text {diff }} \gg v_{\max , g}$, where $v_{\max , M}$ and $v_{\text {max }, g}$ are the maximum values for the flow velocity (along the direction of the concentration gradient) due to Marangoni convection and gravity-driven convection, respectively.

\section{Time constraints}

In order to define the "window of opportunity" for a "convection free" diffusion experiment, the various constraints on the processing time have to be considered. These are depicted in Fig. 5, as a function of temperature, for different sample sizes and heating conditions. It should be noted that the levitation of samples larger than $4 \mathrm{~mm}$ diameter would require a reduced gravity environment.

The time before the onset of crystallization, $t_{\text {cryst }}$, represents an upper limit for the duration of the liquid diffusion experiment, so the diffusion time must be shorter than $600 \mathrm{~s}$ at $960 \mathrm{~K}$, shorter than $30 \mathrm{~s}$ at $900 \mathrm{~K}$, and shorter than $200 \mathrm{~s}$ at $740 \mathrm{~K}$, as can be read from the TTT-diagram. On the other hand, a time interval of the order of the thermal relaxation time is required before the start of the diffusion experiment to achieve a constant temperature. Using Eq. (10) with the values for $c_{P}$ and $\kappa$ listed in Table $\mathrm{I}, t_{\text {therm }}=15 \mathrm{~ms}$ for a spherical sample of radius $R=1 \mathrm{~mm}$, and even for $R=5 \mathrm{~mm} t_{\text {therm }}=0.4 \mathrm{~s}$ is negligible. Time must also be allocated to quench the sample at the end of the experiment. Because the diffusivity changes rapidly with temperature, the time constant $t_{\text {quench }}$ with which the diffusivity decreases when the temperature is decreasing with rate $T / t_{\text {rad }}$ is

$$
t_{\text {quench }}=t_{\text {rad }} E_{\text {act }} / k_{B} T,
$$

where $E_{\text {act }}$ is the apparent activation energy for diffusion and $k_{B}$ is the Boltzmann constant. Thus, $t_{\text {quench }}$ is the absolute minimum duration of a diffusion experiment.

The time window is further constrained by the requirements of tracer deposition and concentration profile measurement techniques. The inequalities expressing that the timedependent diffusion profile depth $\lambda$ should be larger than $30 \mathrm{~nm}$, but smaller than $1 / 10$ th of the sample radius, can be algebraically manipulated to yield a pair of inequalities involving the diffusion time. Similarly, because the diffusion velocity decreases over time, the lower bounds on $v_{\text {diff }}$ to avoid perturbation of the diffusion profile by Marangoni and buoyancy-driven convective flow are equivalent to an upper bound on the diffusion time. Marangoni convection dominates, since the dynamic bond number $\left.B o\right|_{R<2 \mathrm{~mm}}<1$ for sample sizes small enough to be levitated in standard earth gravity, and in microgravity $B o \ll 1$ for any realistic sample size. For the purpose of plotting Fig. 5, the constraint due to convection was evaluated numerically by using the secant method to search for the value of time where $v_{\text {diff,min }}=100$ $v_{\max , M}$.

When all these constraints are plotted together on a timetemperature diagram as in Fig. 5, a "window of opportunity" for successful liquid diffusion experiments is apparent, with boundaries which depend on the sample size, material properties, and heating configuration. Convection is reducedmoving the corresponding boundary to higher temperatures or longer times-by using a smaller sample, broadening the heating laser beam, or using a symmetric arrangement of multiple heating laser beams.

In the deeply undercooled liquid, the limiting constraints are the radiative cooling rate and the time to crystallization, so the window is enlarged by using a smaller sample. At these temperatures, convective flow is limited by the high 


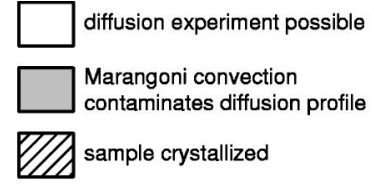

Ediffusion profile reaches sample center

|ा|| diffusion profile too steep to analyse

MIV time too short to quench by radiative cooling
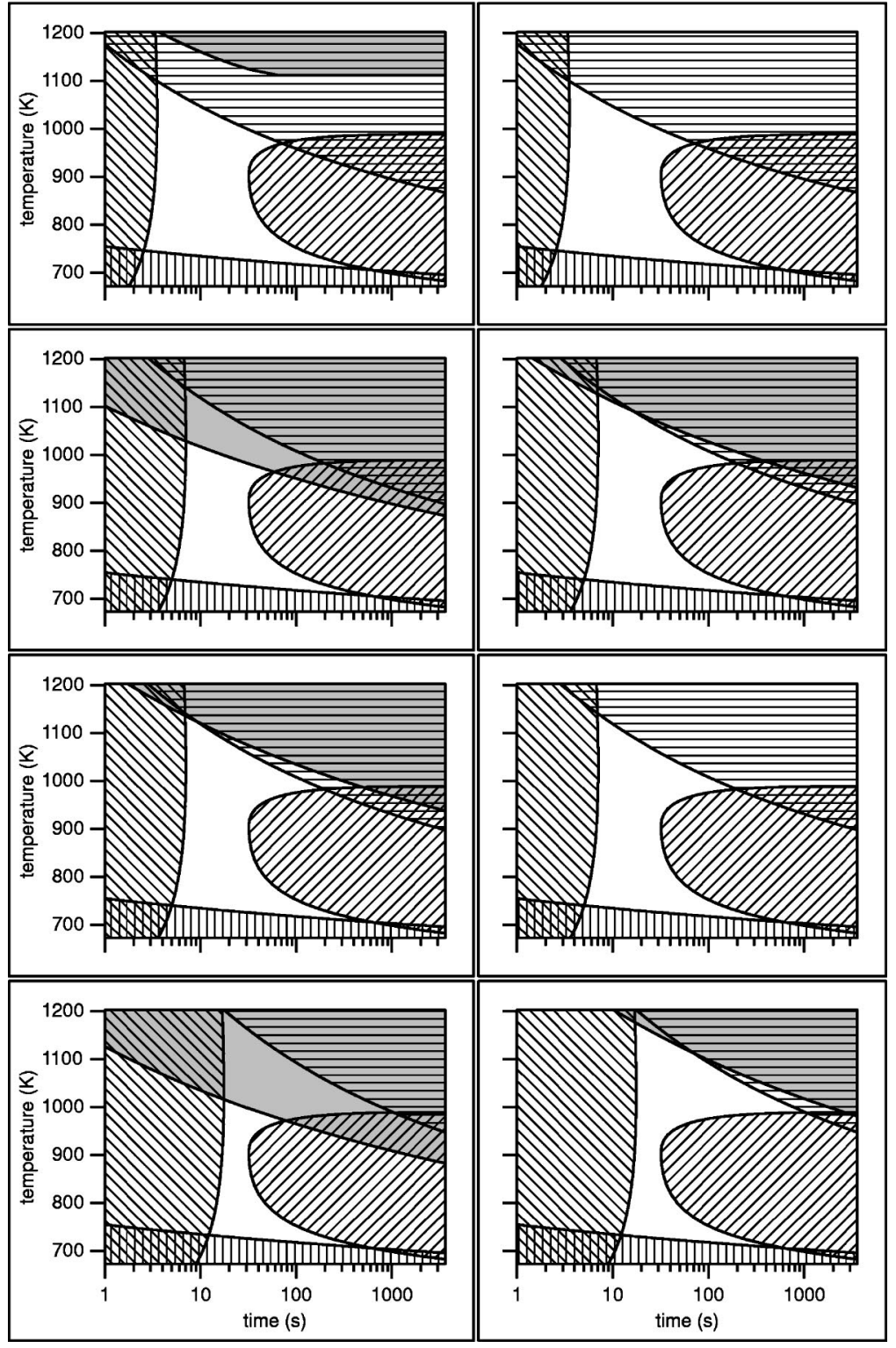

FIG. 5. Time constraints on a diffusion experiment, for different sample sizes and heating conditions. The unshaded area represents experiment duration and temperature combinations where a successful liquid diffusion experiment is possible. See text for an explanation of the different constraints. Top row: $1 \mathrm{~mm}$ sample radius with $0.5 \mathrm{~mm}$ FWHM Gaussian laser intensity profile, 2nd row: $2 \mathrm{~mm}$ sample radius with same (narrow) laser intensity profile, 3rd row: $2 \mathrm{~mm}$ sample radius with laser broadened to $2 \mathrm{~mm}$ FWHM, bottom row: $5 \mathrm{~mm}$ sample radius with laser broadened to $5 \mathrm{~mm}$ FWHM, in each case for a single laser beam (left column) and 4 laser beams in a tetrahedral geometry (right column). viscosity of the alloy, and diffusion experiments should be possible even with a single narrow heating laser beam.

At high temperatures, it becomes important to reduce convective flow. The temperature range where convection can be avoided can be extended upwards significantly, by switching from a single heating laser beam to a tetrahedral arrangement with broadened beams. There is a maximum temperature above which the proposed approach ceases to be useful because the depth of the diffusion profile becomes comparable to the size of the sample before the sample can be quenched. For the Vit106 alloy with samples sizes small enough to be levitated in standard earth gravity, the combination of tetrahedral heating and beam broadening is more than sufficient to move the boundary for contamination of the diffusion profile by convective flow beyond this fundamental constraint. Diffusion experiments should be possible up to approximately the liquidus temperature. Larger samples can be levitated in microgravity. This allows diffusion measurements at still higher temperatures, but also aggravates contamination by convective flow.

Thus, the approach of electrostatic levitation combined with uniform sample heating is effective in reducing convection in a high-temperature reactive liquid sample, thereby enabling diffusion measurements throughout the undercooled liquid range. The approach is also useful for other measurements on high-temperature reactive liquids where a convection-free sample with uniform temperature and containerless processing are desirable.

\section{ACKNOWLEDGMENTS}

This work was supported by the National Aeronautics and Space Administration (Grant No. NAG8-1744). S.B. was 
supported by the EU RTN-network on bulk metallic glasses (HPRN-CT-2000-00033) and by the Belgian Science Policy Office.

${ }^{1}$ W.-K. Rhim, S. K. Chung, D. Barber, K. F. Man, G. Gutt, A. J. Rulison, and R. E. Spjut, Rev. Sci. Instrum. 64, 2961 (1993).

${ }^{2}$ T. Meister, H. Werner, G. Lohoefer, D. M. Herlach, and H. Unbehauen, Control Eng. Pract. 11, 117 (2003).

${ }^{3}$ A. J. Rulison and W.-K. Rhim, Rev. Sci. Instrum. 65, 695 (1994).

${ }^{4}$ M. B. Robinson, D. Li, J. R. Rogers, R. W. Hyers, L. Savage, and T. J. Rathz, Appl. Phys. Lett. 77, 3266 (2000).

${ }^{5}$ T. Ishikawa, P.-F. Paradis, T. Itami, and S. Yoda, J. Chem. Phys. 118, 7912 (2003).

${ }^{6}$ Y. J. Kim, R. Busch, W. L. Johnson, A. J. Rulison, and W.-K. Rhim, Appl. Phys. Lett. 68, 1057 (1996).

${ }^{7}$ C. W. Morton, W. H. Hofmeister, R. J. Bayuzick, A. J. Rulison, and J. L. Watkins, Acta Mater. 46, 6033 (1998).

${ }^{8}$ S. K. Chung, D. B. Thiessen, and W.-K. Rhim, Rev. Sci. Instrum. 67, 3175 (1996).

${ }^{9}$ P.-F. Paradis, T. Ishikawa, and S. Yoda, Appl. Phys. Lett. 83, 4047 (2003).

${ }^{10}$ W.-K. Rhim and T. Ishikawa, Rev. Sci. Instrum. 69, 3628 (1998).

${ }^{11}$ W.-K. Rhim, K. Ohsaka, P.-F. Paradis, and R. E. Spjut, Rev. Sci. Instrum. 70, 2796 (1999).

${ }^{12}$ K. F. Kelton, G. W. Lee, A. K. Gangopadhyay, R. W. Hyers, T. J. Rathz, J. R. Rogers, M. B. Robinson, and D. S. Robinson, Phys. Rev. Lett. 90, 195504 (2003).

${ }^{13}$ P.-F. Paradis, T. Ishikawa, and S. Yoda, J. Non-Cryst. Solids 312, 309 (2002).

${ }^{14}$ H. F. Bauer, Appl. Math. Mech. 65, 461 (1985).

${ }^{15}$ H. F. Bauer, Ingenieria, Mexico 52, 263 (1982).

${ }^{16}$ Thus, Bauer's series expansion for Marangoni flow converges only if the multipole expansion for temperature converges.

${ }^{17}$ The dynamic Bond number differs from the usual definition of the Bond number in that both density and surface tension are differentiated with respect to temperature.

${ }^{18}$ J. Schroers, S. Bossuyt, W.-K. Rhim, J. Li, Z. Zhou, and W. L. Johnson, Rev. Sci. Instrum. 75, 4523 (2004).

${ }^{19}$ W.-K. Rhim and P.-F. Paradis, Rev. Sci. Instrum. 70, 4652 (1999).

${ }^{20}$ L. J. Lewis, Phys. Rev. B 44, 4245 (1991).

${ }^{21}$ U. Geyer, S. Schneider, W. L. Johnson, Y. Qiu, T. A. Tombrello, and M.-P. Macht, Phys. Rev. Lett. 75, 2364 (1995).

${ }^{22}$ K. Knorr, M.-P. Macht, K. Freitag, and H. Mehrer, J. Non-Cryst. Solids 250, 669 (1999)

${ }^{23}$ P. Lehmann, T. Alboussiere, R. Moreau, and J. Uspenski, J. Chim. Phys. Phys.-Chim. Biol. 6, 1105 (1996).

${ }^{24}$ L. B. Jalbert, F. Rosenberger, and R. M. Banish, J. Phys.: Condens. Matter 10, 7113 (1998).

${ }^{25}$ J. I. D. Alexander, J. F. Ramus, and F. Rosenberger, Microgravity Sci. Technol. 9, 158 (1996).

${ }^{26}$ S. Yasuhiro, T. Sato, N. Imaishi, A. Hirata, and M. Kumagawa, Microgravity Sci. Technol. 9, 237 (1996)

${ }^{27}$ G. Mathiak, A. Griesche, K. H. Kraatz, and G. Frohberg, J. Non-Cryst. Solids 207, 412 (1996).

${ }^{28}$ C. C. Hays, J. Schroers, U. Geyer, S. Bossuyt, N. Stein, and W. L. Johnson, Mater. Sci. Forum 343-3, 103 (2000).

${ }^{29}$ C. C. Hays, J. Schroers, T. J. Rathz, R. W. Hyers, J. R. Rogers, M. B. Robinson, and W. L. Johnson, Appl. Phys. Lett. 79, 1605 (2001).

${ }^{30}$ S. Mukerjee, J. Schroers, Z. Zhou, W. L. Johnson, and W.-K. Rhim, Acta Mater. 52, 3689 (2004).

${ }^{31}$ A. L. Greer, Nature (London) 402, 132 (1999).

${ }^{32}$ S. C. Glade, R. Busch, D. S. Lee, W. L. Johnson, R. K. Wunderlich, and H.-J. Fecht, J. Appl. Phys. 87, 7242 (2000).

${ }^{33}$ W. L. Johnson, J. Schroers, U. Geyer, and C. C. Hays, SRD, NASA (1999).

${ }^{34}$ S. Mukherjee, W. L. Johnson, and W.-K. Rhim, Appl. Phys. Lett. 86, 014104 (2005). 